\title{
Sharp bounds for cyclic sums of the ratio of the exradius to the sides of a triangle
}

\author{
Shan-He Wu' ${ }^{1}$ Huan-Peng Yue and Yu-Ming Chu ${ }^{2 *}$
}

"Correspondence:

chuyuming2005@126.com

${ }^{2}$ School of Mathematics and

Computation Science, Hunan City

University, Yiyang, Hunan 413000,

P.R. China

Full list of author information is

available at the end of the article

\begin{abstract}
In this paper, sharp bounds for cyclic sums of the ratio of the exradius to the sides of a triangle are established depending on the circumradius and inradius of the triangle. The best possible parameters for the expressions of bounds are derived. Moreover, an alternative bound for the ratio of the exradius to the sides of triangle, expressed by trigonometric functions, is also considered.
\end{abstract}

MSC: $51 \mathrm{M} 16 ; 26 \mathrm{D} 05 ; 26 \mathrm{D} 15$

Keywords: sharp bounds; triangle; inequality; exradius; ratio-type

\section{Introduction}

For a given triangle $A B C$, we assume that $A, B, C$ denote its angles, $a, b, c$ denote the lengths of its corresponding sides, $s, R$, and $r$ denote the semiperimeter, circumradius, and inradius of a triangle, respectively. Let $w_{a}, w_{b}, w_{c}$ and $m_{a}, m_{b}, m_{c}$ denote respectively the angle bisectors and the medians emanating from vertices $A, B, C$, and let $r_{a}, r_{b}, r_{c}$ denote the exradii tangent to the corresponding sides. In addition, we will customarily use the symbol of cyclic sums such as

$$
\sum f(a)=f(a)+f(b)+f(c), \quad \sum f(a, b)=f(a, b)+f(b, c)+f(c, a) .
$$

The inequalities related to the angle bisectors, medians, exradii, and the sides of a triangle have attracted the interest of many geometers and have motivated a large number of research papers; see e.g. Shi and Wu [1, 2], Jiang [3], Jiang and Bencze [4], Srivastava $e t$ al. [5], Satnoianu [6], Bencze et al. [7-10], Zhelev [11], and Wu et al. [12-16]. An excellent survey on these inequalities can be found in the well-known monographs [17] and [18]. In this paper, we investigate a type of inequality concerning the ratios of the exradii to the sides of a triangle.

We begin by recalling some related results reported in the foregoing literature.

In 1995, Yang [19] and Ma and Dong [20] established the following ratio-type inequality involving the angle bisectors and the sides of a triangle:

$$
\frac{3 \sqrt{3}}{2}+\left(\frac{8}{3}-\frac{3 \sqrt{3}}{2}\right)\left(1-\frac{2 r}{R}\right) \leq \sum \frac{w_{a}}{a} \leq \frac{3 \sqrt{3}}{2}+2 \sqrt{3}\left(\frac{R}{2 r}-1\right) .
$$

(c) 2015 Wu et al. This article is distributed under the terms of the Creative Commons Attribution 4.0 International License (http://creativecommons.org/licenses/by/4.0/), which permits unrestricted use, distribution, and reproduction in any medium, provided you give appropriate credit to the original author(s) and the source, provide a link to the Creative Commons license, and indicate if changes were made. 
In 2000, Chu and Yang [21] presented the following inequality of ratio type associated with the medians and the sides of a triangle:

$$
\frac{3 \sqrt{3}}{2} \sqrt{\frac{7}{3}-\frac{16 R r+4 r^{2}}{s^{2}}} \leq \sum \frac{m_{a}}{a} \leq \sqrt{\frac{r}{R}+\frac{R}{r}+\frac{r^{2}}{R^{2}}+\frac{R^{2}}{r^{2}}} .
$$

Motivated by the above results, the main purpose of this paper is to establish a ratio-type inequality involving the exradii and the sides of a triangle. We present the best possible parameters $\alpha_{1}, \beta_{1}$ and $\alpha_{2}, \beta_{2}$ such that the double inequalities

$$
\begin{aligned}
& \sqrt{\alpha_{1}+\left(\beta_{1}+\left(27-4 \alpha_{1}-2 \beta_{1}\right) \frac{r}{R}\right) \frac{r}{R}} \\
& \leq \sum \frac{r_{a}}{a} \leq \sqrt{\alpha_{2}+\left(\beta_{2}+\left(\frac{27}{2}-2 \alpha_{2}-4 \beta_{2}\right) \frac{r^{2}}{R^{2}}\right) \frac{R}{r}}
\end{aligned}
$$

hold. Moreover, in Section 3.2, we present alternative bounds for the ratios of the exradii to the sides of triangle, in which the bounds are expressed by trigonometric functions.

\section{Lemmas}

In order to prove our main results, we first introduce a lemma. The results stated in the following lemma were established by way of geometric interpretation of the Blundon inequality (see $\mathrm{Wu}$ and $\mathrm{Chu}$ [22] for details), which provides a useful method for proving the inequalities concerning sides, circumradius, and inradius of a triangle.

Lemma 2.1 ([22]) Let $R, r$, and s denote respectively the circumradius, inradius, and semiperimeter of the triangle. Then:

(i) The inequality

$$
s \geq f(R, r)
$$

holds for any triangle if and only if inequality (4) is valid for the isosceles triangles with the vertex angle greater than or equal to $\pi / 3$.

(ii) The inequality

$$
s \leq f(R, r)
$$

holds for any triangle if and only if inequality (5) is valid for the isosceles triangles with the vertex angle less than or equal to $\pi / 3$.

Remark 2.2 ([22]) If the inequality under consideration is homogeneous with respect to $R, r$, and $s$, for convenience of computing, without loss of generality, we may assume that the side lengths of the isosceles triangles are of the form

$$
a=2, \quad b=\frac{1+t^{2}}{1-t^{2}}, \quad c=\frac{1+t^{2}}{1-t^{2}} \quad(0<t<1) .
$$


In fact, under this assumption on the side lengths of the isosceles triangles, the expressions of $s, r$, and $R$ are all rational functions, i.e., we have

$$
\begin{aligned}
& s=\frac{a+b+c}{2}=\frac{2}{1-t^{2}}, \\
& r=\sqrt{\frac{(b+c-a)(c+a-b)(a+b-c)}{4(a+b+c)}}=t \\
& R=\frac{a b c}{\sqrt{(a+b+c)(b+c-a)(c+a-b)(a+b-c)}}=\frac{\left(1+t^{2}\right)^{2}}{4 t\left(1-t^{2}\right)} .
\end{aligned}
$$

This makes calculations much easier.

\section{Main results}

\subsection{Sharp bounds for $\sum \frac{r_{a}}{a}$ in terms of circumradius and inradius of a triangle}

Theorem 3.1 In any triangle ABC, the following double inequalities hold:

$$
\sqrt{\frac{25}{4}+\left(\frac{5}{4}-\frac{r}{2 R}\right) \frac{r}{R}} \leq \sum \frac{r_{a}}{a} \leq \sqrt{\frac{13}{4}+\left(1+\frac{3 r^{2}}{R^{2}}\right) \frac{R}{r}}
$$

with the equalities if and only if the triangle is equilateral. Furthermore, $\frac{25}{4}, \frac{5}{4}, \frac{13}{4}$, and 1 are the best coefficients in (7).

Proof From the formulas of the exradii

$$
r_{a}=\frac{s r}{s-a}, \quad r_{b}=\frac{s r}{s-b}, \quad r_{c}=\frac{s r}{s-c}
$$

and the identities (see [18])

$$
\begin{aligned}
& (s-a)(s-b)(s-c)=s r^{2}, \quad a b c=4 s R r, \\
& \sum a b=s^{2}+4 R r+r^{2}
\end{aligned}
$$

we have

$$
\begin{aligned}
\sum \frac{r_{a}}{a} & =\sum \frac{s r}{a(s-a)} \\
& =\frac{s r}{a b c(s-a)(s-b)(s-c)} \sum a b(s-a)(s-b) \\
& =\frac{1}{4 s R r^{2}}\left(\sum a^{2} b^{2}+s^{2} \sum a b-s \sum a b(a+b)\right) \\
& =\frac{1}{4 s R r^{2}}\left(\left(\sum a b\right)^{2}+s^{2} \sum a b-2 s^{2} \sum a b-s a b c\right) \\
& =\frac{1}{4 s R}\left(s^{2}+16 R^{2}+8 R r+r^{2}\right) \\
& =\frac{1}{4 R}\left(s+\frac{16 R^{2}+8 R r+r^{2}}{s}\right) .
\end{aligned}
$$


Thus, inequality (7) is equivalent to

$$
\begin{aligned}
4 R \sqrt{\frac{25}{4}+\left(\frac{5}{4}-\frac{r}{2 R}\right) \frac{r}{R}} & \leq s+\frac{16 R^{2}+8 R r+r^{2}}{s} \\
& \leq 4 R \sqrt{\frac{13}{4}+\left(1+\frac{3 r^{2}}{R^{2}}\right) \frac{R}{r}}
\end{aligned}
$$

By the Euler inequality $R \geq 2 r$ and the Gerretsen's inequality (see [17])

$$
\sqrt{16 R r-5 r^{2}} \leq s \leq \sqrt{4 R^{2}+4 R r+3 r^{2}}
$$

it is easy to verify that the function

$$
g(s)=s+\frac{16 R^{2}+8 R r+r^{2}}{s}
$$

is strictly decreasing on the interval $\left[\sqrt{16 R r-5 r^{2}}, \sqrt{4 R^{2}+4 R r+3 r^{2}}\right]$. Hence, inequality (8) can be equivalently transformed to the form

$$
f_{1}(R, r) \leq s \leq f_{2}(R, r)
$$

By Lemma 2.1, in order to prove inequality (8) for any triangle, it suffices to prove inequality (8) for the isosceles triangle. Note that inequality (8) is homogeneous with respect to $R, r$, and $s$, so that we may assume that the side lengths of the triangle are

$$
a=2, \quad b=\frac{1+t^{2}}{1-t^{2}}, \quad c=\frac{1+t^{2}}{1-t^{2}}
$$

for some $0<t<1$. Further, the semiperimeter $s$, inradius $r$, and the circumradius $R$ of the triangle can be expressed as follows:

$$
s=\frac{2}{1-t^{2}}, \quad r=t, \quad R=\frac{\left(1+t^{2}\right)^{2}}{4 t\left(1-t^{2}\right)}
$$

Substituting $t$ for $s, r, R$ gives

$$
\begin{aligned}
(s+ & \left.\frac{16 R^{2}+8 R r+r^{2}}{s}\right)^{2}-16 R^{2}\left(\frac{25}{4}+\left(\frac{5}{4}-\frac{r}{2 R}\right) \frac{r}{R}\right) \\
= & \left(\frac{2}{1-t^{2}}+\frac{1-t^{2}}{2}\left(\frac{\left(1+t^{2}\right)^{4}}{t^{2}\left(1-t^{2}\right)^{2}}+\frac{2\left(1+t^{2}\right)^{2}}{\left(1-t^{2}\right)}+t^{2}\right)\right)^{2} \\
& -\frac{\left(1+t^{2}\right)^{4}}{t^{2}\left(1-t^{2}\right)^{2}}\left(\frac{25}{4}+\left(\frac{5}{4}-\frac{2 t^{2}\left(1-t^{2}\right)}{\left(1+t^{2}\right)^{2}}\right) \frac{4 t^{2}\left(1-t^{2}\right)}{\left(1+t^{2}\right)^{2}}\right) \\
= & \frac{1}{4 t^{4}}\left(3 t^{2}+1\right)\left(3 t^{2}-1\right)^{2} \\
\geq & 0
\end{aligned}
$$


and

$$
\begin{aligned}
(s+ & \left.\frac{16 R^{2}+8 R r+r^{2}}{s}\right)^{2}-16 R^{2}\left(\frac{13}{4}+\left(1+\frac{3 r^{2}}{R^{2}}\right) \frac{R}{r}\right) \\
= & \left(\frac{2}{1-t^{2}}+\frac{1-t^{2}}{2}\left(\frac{\left(1+t^{2}\right)^{4}}{t^{2}\left(1-t^{2}\right)^{2}}+\frac{2\left(1+t^{2}\right)^{2}}{\left(1-t^{2}\right)}+t^{2}\right)\right)^{2} \\
& -\frac{\left(1+t^{2}\right)^{4}}{t^{2}\left(1-t^{2}\right)^{2}}\left(\frac{13}{4}+\left(1+\frac{48 t^{4}\left(1-t^{2}\right)^{2}}{\left(1+t^{2}\right)^{4}}\right) \frac{\left(1+t^{2}\right)^{2}}{4 t^{2}\left(1-t^{2}\right)}\right) \\
= & \frac{1}{\left(t^{2}-1\right)^{3}}\left(3 t^{2}-1\right)^{2}\left(t^{2}+1\right)^{2} \\
\leq & 0 .
\end{aligned}
$$

Hence, inequality (7) holds for the isosceles triangle. We thus deduce from Lemma 2.1 that inequality (7) is valid for any triangle. Additionally, note that the equality holds in the above inequalities if and only if $t=\frac{1}{\sqrt{3}}$, that is, when the triangle is equilateral.

Next, we need to show that the coefficients $\frac{25}{4}, \frac{5}{4}, \frac{13}{4}$ and 1 , are the best possible in the strong sense.

Consider inequality (7) in a general form as

$$
\begin{aligned}
& \sqrt{\alpha_{1}+\left(\beta_{1}+\left(27-4 \alpha_{1}-2 \beta_{1}\right) \frac{r}{R}\right) \frac{r}{R}} \\
& \leq \sum \frac{r_{a}}{a} \leq \sqrt{\alpha_{2}+\left(\beta_{2}+\left(\frac{27}{2}-2 \alpha_{2}-4 \beta_{2}\right) \frac{r^{2}}{R^{2}}\right) \frac{R}{r}}
\end{aligned}
$$

Putting

$$
a=2, \quad b=\frac{1+t^{2}}{1-t^{2}}, \quad c=\frac{1+t^{2}}{1-t^{2}} \quad(0<t<1)
$$

in the left-hand side inequality of (11) yields

$$
\begin{gathered}
\alpha_{1}+\left(\beta_{1}+\left(27-4 \alpha_{1}-2 \beta_{1}\right) \frac{4 t^{2}\left(1-t^{2}\right)}{\left(1+t^{2}\right)^{2}}\right) \frac{4 t^{2}\left(1-t^{2}\right)}{\left(1+t^{2}\right)^{2}} \\
\leq \frac{t^{2}\left(1-t^{2}\right)^{2}}{\left(1+t^{2}\right)^{4}}\left(\frac{2}{1-t^{2}}+\frac{1-t^{2}}{2}\left(\frac{\left(1+t^{2}\right)^{4}}{t^{2}\left(1-t^{2}\right)^{2}}+\frac{2\left(1+t^{2}\right)^{2}}{\left(1-t^{2}\right)}+t^{2}\right)\right)^{2} \\
\Longleftrightarrow \quad\left(3 t^{2}-1\right)^{2}\left(28 t^{6} \alpha_{1}-40 t^{4} \alpha_{1}-4 t^{2} \alpha_{1}-16 t^{4} \beta_{1}+16 t^{6} \beta_{1}\right. \\
\left.+26 t^{2}+265 t^{4}-192 t^{6}+1\right) \geq 0 .
\end{gathered}
$$

Taking the limit in (12) as $t \rightarrow 1$, we get $\alpha_{1} \leq \frac{25}{4}$. Then, substituting the coefficient of $\alpha_{1}=\frac{25}{4}$ into (12), we have

$$
\left(3 t^{2}-1\right)^{2}\left(16 t^{4} \beta_{1}-17 t^{4}-2 t^{2}-1\right) \leq 0 .
$$

Letting $t \rightarrow 1$ in (13) yields $\beta_{1} \leq \frac{5}{4}$. 
Consequently, the coefficients $\alpha_{1}=\frac{25}{4}$ and $\beta_{1}=\frac{5}{4}$ are the best possible in the left-hand side inequality of (11), that is, they cannot be replaced by larger constants.

Similarly, putting

$$
a=2, \quad b=\frac{1+t^{2}}{1-t^{2}}, \quad c=\frac{1+t^{2}}{1-t^{2}} \quad(0<t<1)
$$

in the right-hand side inequality of (11), it follows that

$$
\begin{gathered}
\alpha_{2}+\left(\beta_{2}+\left(\frac{27}{2}-2 \alpha_{2}-4 \beta_{2}\right) \frac{16 t^{4}\left(1-t^{2}\right)^{2}}{\left(1+t^{2}\right)^{4}}\right) \frac{\left(1+t^{2}\right)^{2}}{4 t^{2}\left(1-t^{2}\right)} \\
\geq \frac{t^{2}\left(1-t^{2}\right)^{2}}{\left(1+t^{2}\right)^{4}}\left(\frac{2}{1-t^{2}}+\frac{1-t^{2}}{2}\left(\frac{\left(1+t^{2}\right)^{4}}{t^{2}\left(1-t^{2}\right)^{2}}+\frac{2\left(1+t^{2}\right)^{2}}{\left(1-t^{2}\right)}+t^{2}\right)\right)^{2} \\
\Longleftrightarrow \quad\left(3 t^{2}-1\right)^{2}\left(4 t^{4} \alpha_{2}-4 t^{2} \alpha_{2}-10 t^{2} \beta_{2}+7 t^{4} \beta_{2}-\beta_{2}\right. \\
\left.\quad+23 t^{2}-24 t^{4}+1\right) \leq 0 .
\end{gathered}
$$

Taking the limit in (14) as $t \rightarrow 0$, we obtain $\beta_{2} \geq 1$. Further, substituting the coefficient of $\beta_{2}=1$ into (14) yields

$$
\left(3 t^{2}-1\right)^{2}\left(-4 \alpha_{2}+4 t^{2} \alpha_{2}-17 t^{2}+13\right) \leq 0
$$

Letting $t \rightarrow 0$ in (15) gives $\alpha_{2} \geq \frac{13}{4}$.

Thus, the coefficients $\alpha_{2}=\frac{13}{4}$ and $\beta_{2}=1$ are the best possible in the right-hand side inequality of (11), that is, they cannot be replaced by lesser constants.

The proof of Theorem 3.1 is completed.

Corollary 3.2 In any triangle $A B C$, the following double inequalities hold:

$$
\frac{5}{2}+(3 \sqrt{3}-5) \frac{r}{R} \leq \sum \frac{r_{a}}{a} \leq \frac{4}{\sqrt{3}}+\frac{\sqrt{3}}{12} \cdot \frac{R}{r}
$$

with the equalities if and only if the triangle is equilateral.

Proof According to Theorem 3.1, we have

$$
\sqrt{\frac{25}{4}+\left(\frac{5}{4}-\frac{r}{2 R}\right) \frac{r}{R}} \leq \sum \frac{r_{a}}{a} \leq \sqrt{\frac{13}{4}+\left(1+\frac{3 r^{2}}{R^{2}}\right) \frac{R}{r}} .
$$

On the other hand, from the Euler inequality $R \geq 2 r$ we deduce that

$$
\begin{aligned}
\frac{25}{4} & +\left(\frac{5}{4}-\frac{r}{2 R}\right) \frac{r}{R}-\left(\frac{5}{2}+(3 \sqrt{3}-5) \frac{r}{R}\right)^{2} \\
& =\frac{15 r}{4 R^{2}}(R-2 r)(7-4 \sqrt{3}) \\
& \geq 0
\end{aligned}
$$


and

$$
\begin{aligned}
\frac{13}{4} & +\left(1+\frac{3 r^{2}}{R^{2}}\right) \frac{R}{r}-\left(\frac{4}{\sqrt{3}}+\frac{\sqrt{3}}{12} \cdot \frac{R}{r}\right)^{2} \\
& =-\frac{1}{48 R r^{2}}(R-2 r)\left((R-7 r)^{2}+23 r^{2}\right) \\
& \leq 0,
\end{aligned}
$$

which leads to the desired inequalities in Corollary 3.2.

Remark 3.3 In the left-hand side inequality of (16), the constant $\frac{5}{2}$ is the best possible. In fact, putting

$$
a=2, \quad b=\frac{1+t^{2}}{1-t^{2}}, \quad c=\frac{1+t^{2}}{1-t^{2}} \quad(0<t<1)
$$

in the inequality

$$
\mu+(3 \sqrt{3}-2 \mu) \frac{r}{R} \leq \sum \frac{r_{a}}{a}
$$

and taking the limit as $t \rightarrow 1$, it follows that $\mu \leq \frac{5}{2}$. Thus, the best possible value for constant $\mu$ in (18) should be $\mu=\frac{5}{2}$.

In the same way as in the proof of Theorem 3.1, we can determine the best constant for the right-hand side inequality of (16), i.e., the best constant $\lambda$ for the inequality

$$
\sum \frac{r_{a}}{a} \leq \lambda+\left(\frac{3 \sqrt{3}}{4}-\frac{\lambda}{2}\right) \frac{R}{r}
$$

is

$$
\lambda=\frac{\sqrt{3} t_{0}^{4}+26 t_{0}^{3}+20 \sqrt{3} t_{0}^{2}+10 t_{0}+3 \sqrt{3}}{6 t_{0}^{4}+4 \sqrt{3} t_{0}^{3}+8 t_{0}^{2}+4 \sqrt{3} t_{0}+2} \approx 2.3278,
$$

where $t_{0}$ is the real root in the interval $\left(0, \frac{1}{\sqrt{3}}\right)$ of the equation

$$
9 t^{5}+11 \sqrt{3} t^{4}-4 \sqrt{3} t^{2}-9 t+\sqrt{3}=0 .
$$

\subsection{Bounds for $\sum \frac{r_{a}}{a}$ expressed by trigonometric functions}

Theorem 3.4 In any triangle $A B C$, the following double inequalities hold:

$$
2 \cos \frac{\alpha}{2}+\frac{1}{2} \tan \left(\frac{\pi}{4}+\frac{\alpha}{4}\right) \leq \sum \frac{r_{a}}{a} \leq 2 \cos \frac{\beta}{2}+\frac{1}{2} \tan \left(\frac{\pi}{4}+\frac{\beta}{4}\right)
$$

where $\alpha=\min \{A, B, C\}$ and $\beta=\max \{A, B, C\}$. Furthermore, the equalities in (20) hold if and only if the triangle is isosceles.

Proof By using the formulas of exradii (see [18])

$$
r_{a}=4 R \cos \frac{B}{2} \cos \frac{C}{2} \sin \frac{A}{2},
$$




$$
\begin{aligned}
& r_{b}=4 R \cos \frac{C}{2} \cos \frac{A}{2} \sin \frac{B}{2}, \\
& r_{c}=4 R \cos \frac{A}{2} \cos \frac{B}{2} \sin \frac{C}{2},
\end{aligned}
$$

we get

$$
\sum \frac{r_{a}}{a}=\sum \frac{\cos \frac{B}{2} \cos \frac{C}{2}}{\cos \frac{A}{2}}
$$

Without loss of generality, we assume that $A \geq B$ and $A \geq C$. To prove the right-hand side inequality of (20), it suffices to prove that

$$
\sum \frac{r_{a}}{a} \leq 2 \cos \frac{A}{2}+\frac{1}{2} \tan \left(\frac{\pi}{4}+\frac{A}{4}\right)
$$

Direct computation gives

$$
\begin{aligned}
2 & \cos \frac{A}{2}+\frac{1}{2} \tan \left(\frac{\pi}{4}+\frac{A}{4}\right)-\sum \frac{r_{a}}{a} \\
& =2 \cos \frac{A}{2}+\frac{1}{2} \tan \left(\frac{\pi}{4}+\frac{A}{4}\right)-\sum \frac{\cos \frac{B}{2} \cos \frac{C}{2}}{\cos \frac{A}{2}} \\
& =\cos \frac{A}{2}\left(2-\frac{\cos \frac{B}{2}}{\cos \frac{C}{2}}-\frac{\cos \frac{C}{2}}{\cos \frac{B}{2}}\right)+\frac{1+\sin \frac{A}{2}-2 \cos \frac{B}{2} \cos \frac{C}{2}}{2 \cos \frac{A}{2}} \\
& =\frac{-\cos \frac{A}{2}\left(\cos \frac{B}{2}-\cos \frac{C}{2}\right)^{2}}{\cos \frac{B}{2} \cos \frac{C}{2}}+\frac{1-\cos \left(\frac{B}{2}-\frac{C}{2}\right)}{2 \cos \frac{A}{2}} \\
& =\frac{-4 \cos \frac{A}{2} \sin ^{2}\left(\frac{B}{4}+\frac{C}{4}\right) \sin ^{2}\left(\frac{B}{4}-\frac{C}{4}\right)}{\cos \frac{B}{2} \cos \frac{C}{2}}+\frac{\sin ^{2}\left(\frac{B}{4}-\frac{C}{4}\right)}{\cos \frac{A}{2}} \\
& =\frac{\sin ^{2}\left(\frac{B}{4}-\frac{C}{4}\right)}{\cos \frac{A}{2} \cos \frac{B}{2} \cos \frac{C}{2}}\left(\cos \frac{B}{2} \cos \frac{C}{2}-2 \cos ^{2} \frac{A}{2}\left(1-\sin \frac{A}{2}\right)\right) .
\end{aligned}
$$

From $A \geq B, A \geq C$, and $A, B, C \in(0, \pi)$ we conclude that

$$
0<\cos \frac{A}{2} \leq \cos \frac{B}{2}, \quad 0<\cos \frac{A}{2} \leq \cos \frac{C}{2}
$$

and

$$
\sin \frac{A}{2} \geq \sin \frac{\pi}{6}=\frac{1}{2}
$$

Hence,

$$
\cos \frac{B}{2} \cos \frac{C}{2}-2 \cos ^{2} \frac{A}{2}\left(1-\sin \frac{A}{2}\right) \geq \cos ^{2} \frac{A}{2}-2 \cos ^{2} \frac{A}{2}\left(1-\sin \frac{A}{2}\right) \geq 0,
$$

which implies that

$$
2 \cos \frac{A}{2}+\frac{1}{2} \tan \left(\frac{\pi}{4}+\frac{A}{4}\right)-\sum \frac{r_{a}}{a} \geq 0,
$$


and the equality holds if and only if $B=C$. Hence, the right-hand side inequality of (20) is proved.

Similarly to the above, we assume that $C \leq A$ and $C \leq B$. To prove the left-hand side inequality of (20), it suffices to prove that

$$
\sum \frac{r_{a}}{a} \geq 2 \cos \frac{C}{2}+\frac{1}{2} \tan \left(\frac{\pi}{4}+\frac{C}{4}\right) .
$$

After a simple computation, we obtain

$$
\begin{aligned}
2 & \cos \frac{C}{2}+\frac{1}{2} \tan \left(\frac{\pi}{4}+\frac{C}{4}\right)-\sum \frac{r_{a}}{a} \\
= & 2 \cos \frac{C}{2}+\frac{1}{2} \tan \left(\frac{\pi}{4}+\frac{C}{4}\right)-\sum \frac{\cos \frac{B}{2} \cos \frac{C}{2}}{\cos \frac{A}{2}} \\
& =\frac{\sin ^{2}\left(\frac{A}{4}-\frac{B}{4}\right)}{\cos \frac{A}{2} \cos \frac{B}{2} \cos \frac{C}{2}}\left(\cos \frac{A}{2} \cos \frac{B}{2}-2 \cos ^{2} \frac{C}{2}\left(1-\sin \frac{C}{2}\right)\right) .
\end{aligned}
$$

Noting that $C \leq A, C \leq B$, and $A, B, C \in(0, \pi)$, we have

$$
\cos \frac{C}{2} \geq \cos \frac{A}{2}>0, \quad \cos \frac{C}{2} \geq \cos \frac{B}{2}>0
$$

and

$$
\sin \frac{C}{2} \leq \sin \frac{\pi}{6}=\frac{1}{2} .
$$

Thus,

$$
\cos \frac{A}{2} \cos \frac{B}{2}-2 \cos ^{2} \frac{C}{2}\left(1-\sin \frac{C}{2}\right) \leq \cos ^{2} \frac{C}{2}-2 \cos ^{2} \frac{C}{2}\left(1-\sin \frac{C}{2}\right) \leq 0,
$$

which leads us to

$$
\sum \frac{r_{a}}{a} \geq 2 \cos \frac{C}{2}+\frac{1}{2} \tan \left(\frac{\pi}{4}+\frac{C}{4}\right)
$$

with equality if and only if $A=B$. The left-hand side inequality of (20) is proved. This completes the proof of Theorem 3.4.

Remark 3.5 In Theorem 3.4, the expressions of lower and upper bounds involve a common function

$$
\psi(x)=2 \cos \frac{x}{2}+\frac{1}{2} \tan \left(\frac{\pi}{4}+\frac{x}{4}\right), \quad x \in(0, \pi) .
$$

Differentiating $\psi(x)$ with respect to $x$ gives

$$
\begin{aligned}
\psi^{\prime}(x) & =-\sin \frac{x}{2}+\frac{1}{8} \sec ^{2}\left(\frac{\pi}{4}+\frac{x}{4}\right) \\
& =\frac{\left(1-2 \sin \frac{x}{2}\right)^{2}}{4\left(1-\sin \frac{x}{2}\right)} \geq 0 .
\end{aligned}
$$

This means that $\psi(x)$ is increasing on the interval $(0, \pi)$. 
By the monotonicity of the function $\psi(x)$ we can easily deduce the following consequence of Theorem 3.4.

\section{Corollary 3.6}

(i) In any triangle $A B C$, we have

$$
\sum \frac{r_{a}}{a}>\frac{5}{2}
$$

(ii) In an acute triangle $A B C$, we have

$$
\sum \frac{r_{a}}{a}<\frac{1+3 \sqrt{2}}{2}
$$

Proof Note that the function

$$
\psi(x)=2 \cos \frac{x}{2}+\frac{1}{2} \tan \left(\frac{\pi}{4}+\frac{x}{4}\right)
$$

is increasing on the interval $(0, \pi)$. Using Theorem 3.4 together with the facts that

$$
0<\min \{A, B, C\} \leq \frac{\pi}{3} \quad \text { for any triangle }
$$

and

$$
\frac{\pi}{3} \leq \max \{A, B, C\}<\frac{\pi}{2} \quad \text { for an acute triangle, }
$$

we obtain

$$
\sum \frac{r_{a}}{a}>f(0)=\frac{5}{2}
$$

and

$$
\sum \frac{r_{a}}{a}<f\left(\frac{\pi}{2}\right)=\frac{1+3 \sqrt{2}}{2} .
$$

This proves the desired inequalities in Corollary 3.6.

\section{Conclusions}

Let us now give a summary of the contents. First, we established sharp bounds for cyclic sums of the ratio of the exradii to the sides of a triangle, depending on the circumradius and inradius of the triangle, as follows:

$$
\sqrt{\frac{25}{4}+\left(\frac{5}{4}-\frac{r}{2 R}\right) \frac{r}{R}} \leq \sum \frac{r_{a}}{a} \leq \sqrt{\frac{13}{4}+\left(1+\frac{3 r^{2}}{R^{2}}\right) \frac{R}{r}},
$$

where the coefficients $\frac{25}{4}, \frac{5}{4}, \frac{13}{4}$, and 1 are the best possible in the strong sense.

Second, we presented alternative bounds for $\sum \frac{r_{a}}{a}$, in which the bounds are expressed by the trigonometric functions associated with the maximum and minimum angles of a triangle.

Finally, we deduced several consequences from the above-mentioned results. 


\section{Competing interests}

The authors declare that there is no conflict of interests regarding the publication of this article.

\section{Authors' contributions}

All authors contributed equally and significantly in this paper. All authors read and approved the final manuscript.

\section{Author details}

${ }^{1}$ Department of Mathematics, Longyan University, Fujian, 364012, P.R. China. ${ }^{2}$ School of Mathematics and Computation Science, Hunan City University, Yiyang, Hunan 413000, P.R. China.

\section{Acknowledgements}

This research was supported by the Natural Science Foundation of China under Grants 11371125 and 61374086, the Natural Science Foundation of Hunan Province under Grant 14JJ2127, the Natural Science Foundation of Zhejiang Province under Grant LY13A010004, the National Training Programs of China for Undergraduate Innovation and Entrepreneurship under Grant 201411312003, and the Foundation of Scientific Research Project of Fujian Province Education Department under Grant JK2013051.

\section{Received: 29 October 2015 Accepted: 1 December 2015 Published online: 08 December 2015}

\section{References}

1. Shi, S-C, Wu, Y-D: An artificial proof of a geometric inequality in a triangle. J. Inequal. Appl. 2013, Article 329 (2013)

2. Shi, S-C, Wu, Y-D: The best constant in a geometric inequality relating medians, inradius and circumradius in a triangle. J. Math. Inequal. 7(2), 183-194 (2013)

3. Jiang, W-D: An inequality involving the angle bisectors and an interior point of a triangle. Forum Geom. 8, 73-76 (2008)

4. Jiang, W-D, Bencze, M: Some geometric inequalities involving angle bisectors and medians of a triangle. J. Math. Inequal. 5(3), 363-369 (2011)

5. Srivastava, HM, Lokesha, V, Wu, Y-D: A new refinement of the Janous-Gmeiner inequality for a triangle. Comput. Math. Appl. 62(5), 2349-2353 (2011)

6. Satnoianu, RA: General power inequalities between the sides and the circumscribed and inscribed radii related to the fundamental triangle inequality. Math. Inequal. Appl. 5(4), 745-751 (2002)

7. Bencze, M, Wu, S-H: Ratio-type inequalities for bisectors, medians, altitudes, and sides of a triangle. Crux Math. Math. Mayhem 36(5), 304-308 (2010)

8. Bencze, M, Gao, M-Z: Some new inequalities for the bisectors in a triangle. Octogon Math. Mag. 19(2), 353-364 (2011)

9. Bencze, M, Zhao, C-J: Inequalities for medians, altitudes and bisectors in a general triangle. Octogon Math. Mag. 20(1), 158-162 (2012)

10. Bencze, M, Zhao, C-J: About angles between medians and altitudes in a triangle. Octogon Math. Mag. 18(2), 323-333 (2010)

11. Zhelev, Z: One group of inequalities with altitudes, medians and bisectors in triangle. Octogon Math. Mag. 22(1), 35-43 (2014)

12. $\mathrm{Wu}, \mathrm{S}-\mathrm{H}, \mathrm{Zhang}, \mathrm{Z}-\mathrm{H}$ : A class of inequalities related to the angle bisectors and the sides of a triangle. J. Inequal. Pure Appl. Math. 7(3), Article 108 (2006)

13. $\mathrm{Wu}, \mathrm{S}-\mathrm{H}$ : A sharpened version of the fundamental triangle inequality. Math. Inequal. Appl. 11(3), 477-482 (2008)

14. Wu, S-H: An inverse inequality for the angle bisectors and its analogue. J. Suzhou Railw. Teach. Coll. 17(4), 42-44 (2000) (in Chinese)

15. Wu, S-H: A strengthened inequality for the angle bisectors and its analogue. J. Longyan Teach. Coll. 20(6), 98-99 (2002) (in Chinese)

16. $\mathrm{Wu}, \mathrm{S}-\mathrm{H}$ : Some inequalities related to the angle bisectors and the radius of escribed circle. J. Guizhou Educ. Coll. 13(4), 16-18 (2002) (in Chinese)

17. Bottema, O, Djordjević, RZ, Janić, RR, Mitrinović, DS, Vasić, PM: Geometric Inequalities. Wolters-Noordhoff, Groningen (1969)

18. Mitrinović, DS, Pečarić, JE, Volenec, V: Recent Advances in Geometric Inequalities. Kluwer Academic, Dordrecht (1989)

19. Yang, X-Z: Sharpness of an inequality involving the angle bisectors. Middle School Math. 12, $20-22$ (1995) (in Chinese)

20. Ma, T-Y, Dong, W-L: The inverse version of an inequality concerning the angle bisectors. Bull. Math. (Wuhan) 11, 19-20 (1995) (in Chinese)

21. Chu, X-G, Yang, X-Z: Several Inequalities Associated with the Medians of a Triangle. Research in Inequalities. Tibet People's Publishing House, Lasha (2000) (in Chinese)

22. $\mathrm{Wu}, \mathrm{S}-\mathrm{H}, \mathrm{Chu}, \mathrm{Y}-\mathrm{M}$ : Geometric interpretation of Blundon's inequality and Ciamberlini's inequality. J. Inequal. Appl. 2014, Article 381 (2014) 